Колесников Александр Михайлович, Кокодей Татьяна Александровна, Хитущенко Виталина Валерьевна

\title{
ИСПОЛЬЗОВАНИЕ ЭКОНОМИКО-МАТЕМАТИЧЕСКИХ МЕТОДОВ ДЛЯ ОЦЕНКИ РЕЗУЛЬТАТОВ ЭКСПЕРИМЕНТА ПО ВНЕДРЕНИЮ ЭЛЕКТРОННЫХ УЧЕБНЫХ КУРСОВ ПО АНГЛИЙСКОМУ ЯЗЫКУ СДО МОODLЕ В ОБРАЗОВАТЕЛЬНЫЙ ПРОЦЕСС СЕВАСТОПОЛЬСКОГО ГОСУДАРСТВЕННОГО УНИВЕРСИТЕТА
}

В данном исследовании описана организация и проанализированы результаты проведённого проверочного педагогического эксперимента по внедрению в образовательный процесс электронных учебных курсов по англоязычной подготовке студентов на базе Севастопольского государственного университета. Обработка полученных результатов методами главных компонент и дисперсионного анализа показали эффективность данного внедрения с точки зрения повышения успеваемости студентов по английскому языку

Ключевые слова: СДО Моодlе, электронный учебный курс, английский язык.

Alexander Kolesnikov, Tatiana Kokodey, Vitalina Khituschenko

THE USE OF ECONOMIC AND MATHEMATICAL METHODS FOR EVALUATING THE RESULTS OF THE EXPERIMENT

ON THE INTRODUCTION OF AN ENGLISH LANGUAGE E- COURSE IN LMS MOODLE INTO THE EDUCATIONAL PROCESS OF SEVASTOPOL STATE UNIVERSITY

This study describes the organization and analyzes the results of the conducted verification pedagogical experiment on the introduction of e-learning courses on the English-language training of students on the basis of Sevastopol State University into the educational process. The processing of the obtained results by the methods of principal components and analysis of variance showed the effectiveness of this implementation in terms of improving students' academic performance in English.

Keywords: LMS Moodle, e-learning course, English language.

Bведениe / Introduction. В настоящее время практический уровень владения английским языком выпускниками организаций высшего образования не всегда отвечает социальному заказу общества, обусловливая необходимость поиска новых подходов, методов, технологий организации учебного процесса иноязычной профессиональной подготовки в контексте глобальной цифровизации образования.

В тоже время в вузах наблюдается стремительная тенденция создания и использования недостаточно качественных электронных образовательных ресурсов, что может ухудшить уровень языковой подготовки студентов. Кроме того, в попытке вывести образовательный процесс на новый уровень, в организациях высшего образования широко внедряются открытые системы дистанционного обучения, преимущественно СДО Moodle. Зачастую их использование неэффективно по двум основным причинам: 1) неправильное использование многофункционального инструментария системы (из-за её относительной новизны) и 2) сопротивление изменениям профессорско-преподавательского состава. 
В связи с этим возникает необходимость оценки эффективности внедрения разработанных электронных учебных курсов СДО Moodle, в частности по английскому языку.

Анализ существующих исследований. Значительное влияние на становление теории педагогического эксперимента оказали работы таких авторов, как Ч. Хикс [1], Е. В. Яковлев [2], И. А. Степанкин [3], В. А. Штофф [4], Г. И. Батищев [5] и др. Обобщая их определения данного понятия, можно согласиться со следующей трактовкой: «научно поставленный опыт преобразования педагогического процесса в точно учитываемых условиях».

Тем не менее, несмотря на весомые разработки теоретических положений, сегодня в педагогической науке и практике нет однозначного и общепризнанного понимания методологических основ педагогического эксперимента, а также способов его эффективной реализации.

Целью исследования была оценка влияния цифровизации образовательного процесса Севастопольского государственного университета, в частности внедрения электронных учебных курсов по английскому языка в СДО Moodle, на эффективность англоязычной профессиональной подготовки студентов уровня бакалавриата, таким образом, создание предпосылок для дальнейшего развития информационно-образовательной среды университета.

Задачи исследования были следующие:

- сформировать две случайных выборки по 30 студентов третьего курса бакалавриата в каждой, так чтобы были представлены различные направления обучения и различные институты Университета. Первая выборка, контрольная группа, должна состоять из студентов, которые обучаются английскому языку по традиционной методике без применения дистанционных образовательных технологий. Вторая выборка - это экспериментальная группа, в которую должны войти студенты, англоязычная профессиональная подготовка которых с четвёртого семестра проводится с использованием электронного учебного курса по английскому языку в СДО Moodle.

- сформировать набор исходных данных по контрольной и экспериментальной группе из итоговых оценок по английскому языку для каждого студента за каждый из пяти семестров обучения

- провести обработку исходных данных в статистическом пакете ПО Gretl, используя инструментарий дисперсионного анализа (ANOVA) и метода главных компонент (PCA), чтобы определить, оказывает ли обучение английскому языку в электронном курсе СДО Мoodleна успеваемость студентов по данной дисциплине.

- проанализировать полученные результаты и сделать выводы.

Описание выборки эксперимента. Студенты уровня бакалавриат, изучающие английский язык, выбраны случайным образом с различных направлений подготовки. Все студенты на момент проведения эксперимента (10.01.19) обучаются на третьем курсе (начало шестого семестра). Их общее количество - 60 человек, 30 из них входят в контрольную группу, в которой при обучении английскому языку применялись традиционные методы (не работали в системе СДО Moodle), а 30 - в экспериментальную, для которой в четвёртом семестре начал применяться электронный учебный курс Moodle при обучении английскому языку.

Maтериалы и методы / Materials and methods.

1. Метод главных компонент (РСА) [6]. Данный метод был предложен Пирсоном в 1901 году и затем вновь открыт и детально разработан Хоттелингом (1933 г.). РСА применяется, например, для сжатия объёмов хранимой информации и упрощения её интерпретации или сравнения многомерных исследуемых объектов, позволяя снизить размерность исходного признакового пространства $x_{1}, \ldots, x_{p}\left(x_{i}-\right.$ исходный признак) и перейти к новым агрегированным признакам $\boldsymbol{y}_{1}, \ldots, y_{p}$. $\left(y_{j}\right.$ - главная компонента), $\quad p^{\prime}<p$. При этом новые показатели $y_{1}, \ldots, y_{p}$. представляют собой линейные комбинации исходных $x_{1}, \ldots, x_{p}$, коррелированных между собой, формула (1). 


$$
\begin{gathered}
y_{j}(x)=w_{1 j}\left(\frac{x_{1}-\bar{x}_{1}}{\sigma_{1}}\right)+\ldots+w_{p j}\left(\frac{x_{p}-\bar{x}_{p}}{\sigma_{p}}\right) ; \\
\sum_{i=1}^{p} w_{i j}^{2}=1(j=1 \ldots p) ; \\
\sum_{i=1}^{p} w_{i j} w_{i k}=0(j, k=1 \ldots p, j \neq k) ;
\end{gathered}
$$

где $\bar{x}_{j}$ и $\sigma_{j}$ - среднее арифметическое и среднеквадратическое отклонение признака $x_{i}$; $w_{i j}-$ коэффициенты главных компонент, максимизирующие дисперсию $y_{j}$, которые находятся из уравнения $(S-\lambda E) \cdot \vec{w}=0$, имеющее решение, если $|S-\lambda E|=0$, где $S$ - ковариационная (или корреляционная) матрица; $\lambda_{i}-$ собственные числа матрицы $S$ равны дисперсиям проекций множества объектов на оси главных компонент.

Традиционный алгоритм расчёта главных компонент включает переход от исходной матрицы наблюдений $X$ к ковариационной (или корреляционной) матрице $S$ между исходными признаками $x_{1}, \ldots, x_{p}$, далее к расчёту собственных чисел $\lambda_{i}$. Основываясь на наибольших собственных числах, наилучшим образом объясняющих исходное пространство признаков, производится переход к главным компонентам путём определения их коэффициентов $w_{j}=\left(w_{1 j}, \ldots, w_{p j}\right)^{\prime}$, максимизирующих дисперсию проекций множества объектов на оси главных компонент. Таким образом, выбираются только те главные компоненты, изменчивость которых покрывает большую часть изменчивости $x_{1}, \ldots, x_{p}$. Если первая главная компонента покрывает большую часть изменчивости $x_{1}, \ldots, x_{p}$, то она может интерпретироваться как интегральный показатель. Например, интегральный показатель успеваемости студента по дисциплине за весь период обучения, рассчитываемый как первая главная компонента, может строиться на основе индивидуальных показателей его успеваемости за каждый семестр.

2. Дисперсионный анализ (ANOVA). Фундаментальная концепция дисперсионного анализа предложена Фишером в 1920 году. Задача дисперсионного анализа заключается в том, чтобы установить, оказывает ли существенное влияние некоторый качественный фактор(-ы) хі, который имеет $р$ уровней или градаций $x_{i 1} \ldots x_{i p}$ на изучаемый количественный признак у (зависимую переменную).

Например, качественным фактором $x_{i}$ может быть наличие или отсутствие системы дистанционного обучения Moodle в университете, тогда данная переменная будет иметь только два уровня («есть» или «нет»). Зависимой количественной переменной у в этом случае может быть итоговая оценка студента за семестр. Тогда, имея данные об итоговых оценках двух групп студентов, одна из которая обучалась без Moodle, а другая - в данной системе, можно определить, оказывает ли внедрение Moodle влияние на успеваемость.

Для решения задачи дисперсионного анализа исследуется статистическая значимость различия между групповыми средними $\left(\overline{y_{1}}, \overline{y_{2}}\right.$, и т. д. $\left.\overline{y_{n}}\right)$ для каждого уровня фактора $x_{i}$ с помощью сравнения (анализа) дисперсий.

То есть при исследовании статистической значимости различия между средними нескольких групп, мы на самом деле сравниваем дисперсии: «межгрупповую (факторную) дисперсию» $\left(\sigma_{\text {between }}^{2}\right)$, порождаемую воздействием конкретного фактора $x_{i}$, и «внутригрупповую (остаточную) дисперсию» ( $\left.\sigma_{\text {within }}^{2}\right)$, обусловленную случайными причинами. Для проверки гипотезы $H_{0}$ о равенстве средних используют статистический критерий, который (если верна гипотеза $H_{0}$ ) имеет распределение Фишера, формула (2):

$$
F=\sigma_{\text {between }}^{2} / \sigma_{\text {within }}^{2}
$$


Используемое программное обеспечение. Используемый в эксперименте статистический пакет программ GRETL (GNU Regression Econometrics and Time Series Library) [7] представляет собой инструментарий для практической реализации сложных вычислительных процедур моделирования. В 2002 году его автор профессор Аллен Котрелл (США) включил GRETL в проект www.sourceforget.net, делая его общедоступным бесплатным продуктом с возможностью дальнейшей доработки открытых кодов (Open Source - свободным программным обеспечением). Таким образом, данный пакет программ, статистические данные для обработки, учебное пособие и исходный код всех выпущенных версий доступны на Интернет-сайтах http://gretl.sourceforge. netилиһttp://www.kufel.torun.pl.

Возможности программы:

1. Основные описательные статистики.

2. Проверка нормальности распределения, распределение частот случайной величины, распределение плотности вероятностей, определение коэффициентов корреляции и т. д.

3. Предусматривает непосредственный доступ к статистическим таблицам. Пакет Gretl содержит встроенные статистические таблицы для следующих распределений: нормального, t-распределения Стьюдента, F-распределения Фишера, хи-квадрат, Пуассона, биномиального и распределения Дарбина - Уотсона. Существует возможность вычисления критических значений, p-value.

4. Анализ временных рядов (набор методов оценивания обобщённым MHК, модели ARMAX и GARCH, система уравнений авторегрессии (VAR), проверка коинтеграции; построение линии тренда, коррелограммы, периодограммы; проверка единичных корней, моделирование типа ARIMA, а также процедуры десезонализации X-12-ARIMA и TRAMO).

5. Регрессионный анализ (одношаговый метод наименьших квадратов (1MHК), взвешенный МНК, двухшаговый МНК - оценка систем одновременных уравнений, методы оценивания логитовых, пробитовых и тобитовых моделей и нелинейных моделей, и т. д.)

6. Метод главных компонент.

7. Дисперсионный анализ.

8. Экспорт и импорт Gretl- MicrosoftExcel и текстовые редакторы (Notepad и т. д).

9. Построение графиков и др.

Результаты и обсуждение / Results and discussion. На первом этапе сформируем набор исходных данных в ПО Gretl по экспериментальной группе, который состоит из итоговых оценок студентов по английскому языку на пять семестров (рис. 1 и 2).

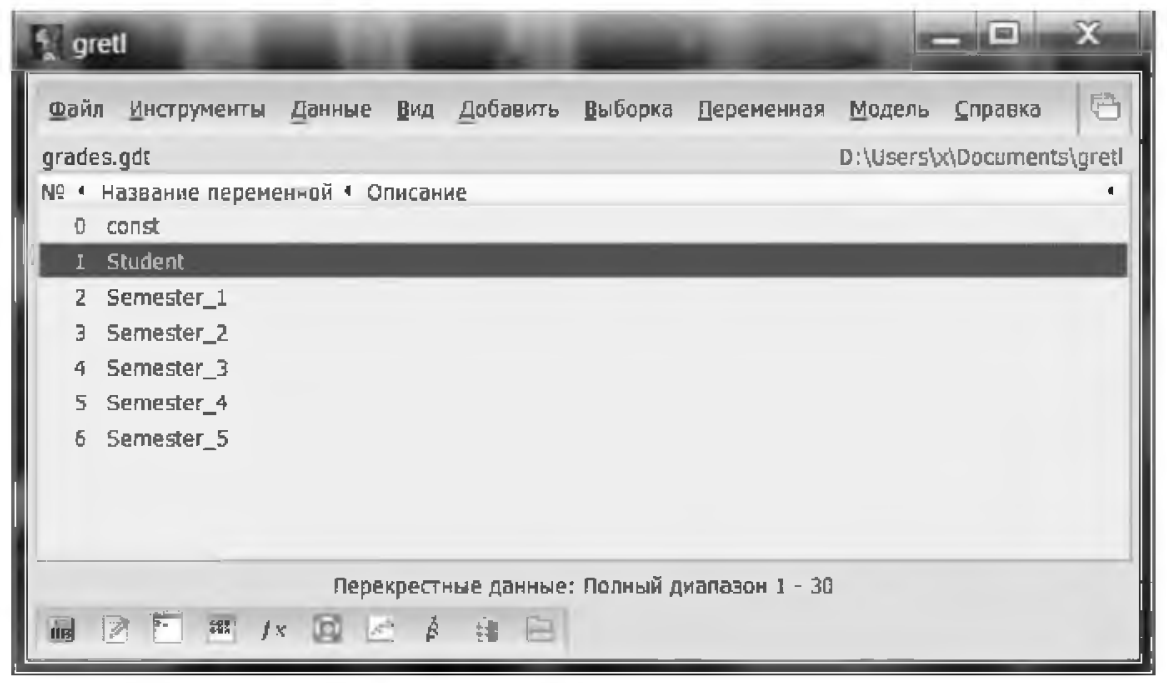

Рис. 1. Набор исходных данных по экспериментальной группе в ПО Gretl 


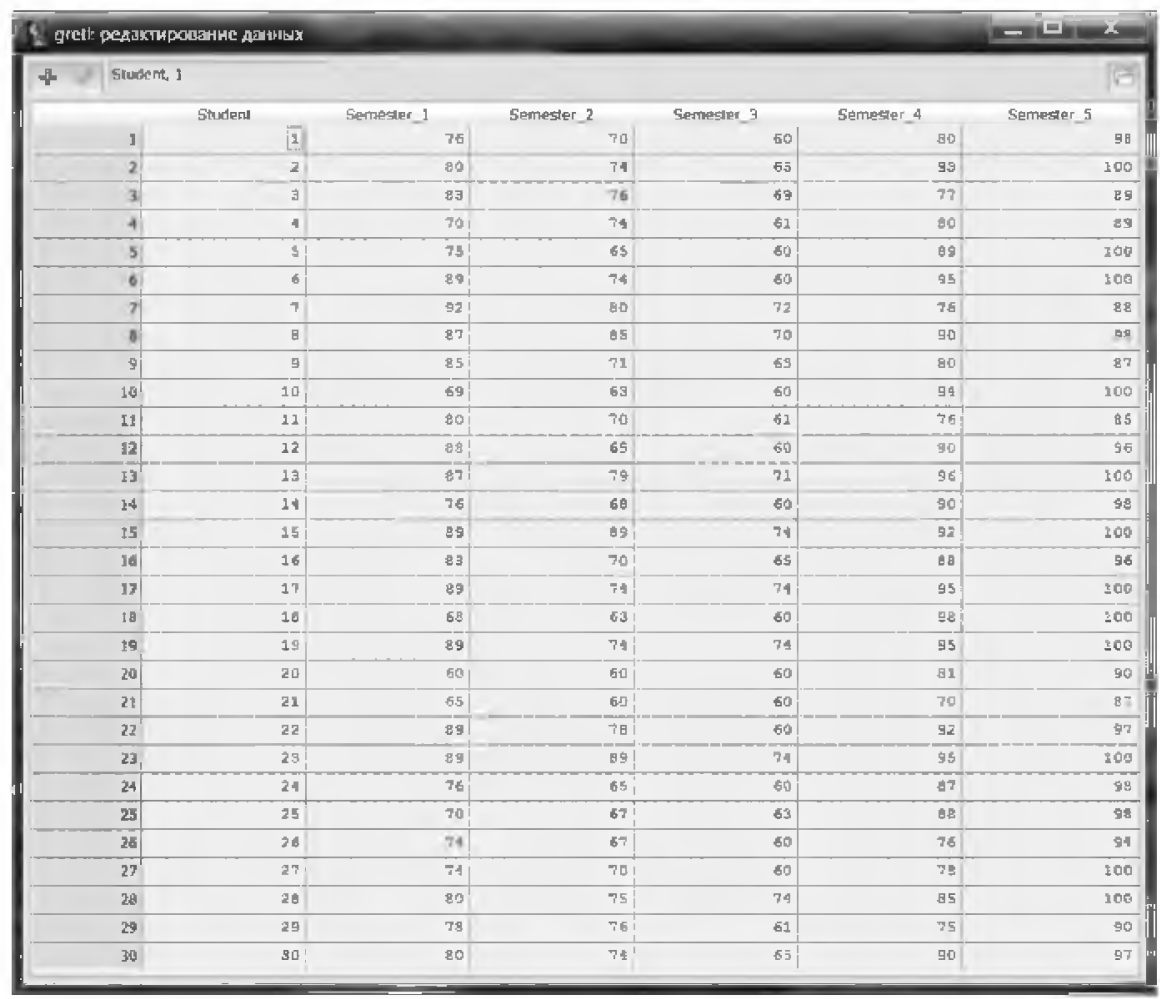

Рис. 2. Просмотр исходных данных ПО Gretl

«Student» - переменная, обозначающая номер студента, а переменные Semester_1... Semester 5 - обозначают итоговые оценки за соответствующие семестры по 100балльной системе.

Эman 2. Поскольку для экспериментальной группы курс Moodle был внедрён в четвёртом семестре, то используем метод главных компонент, чтобы построить два интегральных показателя оценки (рассчитанные как первые главные компоненты): до внедрения Moodle (Y1_1) и после этого (Y2_1).

Y1_1 построим по пространству исходных признаков Semester_1, Semester_2 и Semester_3, учитывая оценки за первые три семестра обучения английскому языку. Реализация этого этапа в программной среде Gretl приведена на рис. 3.

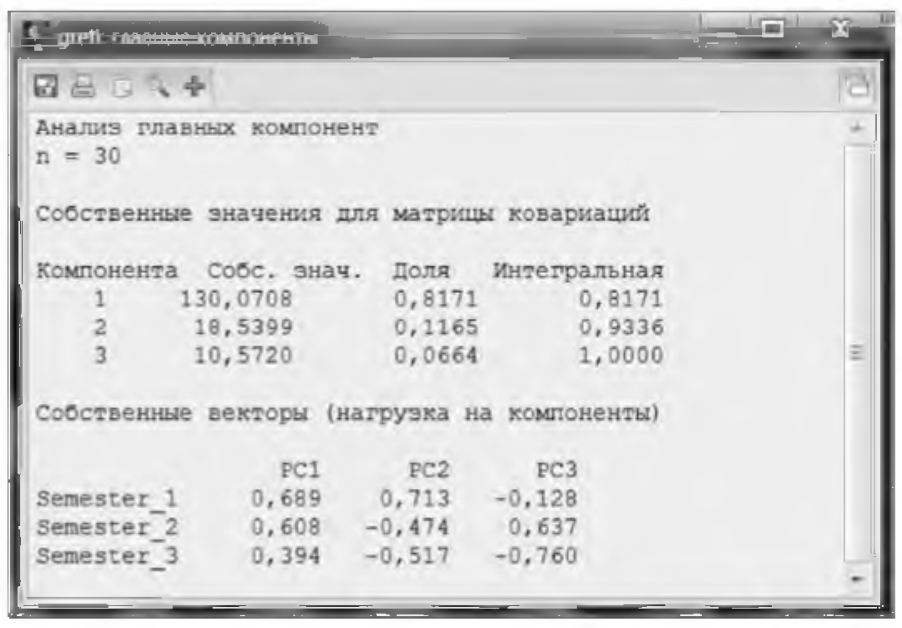

Рис. 3. Окно результатов моделирования интегрального показателя оценки по английскому языку до внедрения Moodle (первые три семестра обучения) 
На рис. 3 первая главная компонента Y1_1 имеет обозначение PC1, она имеет наибольшую значимость и рассматривается как интегральный (обобщённый) показатель оценки:

$$
Y_{1 \_1}=0,689 \cdot \text { Semester }_{1}+0,608 \cdot \text { Semester }_{2}+0,394 \cdot \text { Semester }_{3} \text {. }
$$

Формула показывает зависимость интегрального показателя Y1_1 от исходных признаков Semester 1...Semester 3. Коэффициенты уравнения показывают вклад каждого индивидуального показателя в интегральный.

Столбцы РС $_{i}$ в окне результатов моделирования (рис. 3) содержат значения коэффициентов главных компонент $w_{j}=\left(w_{1 j}, \ldots, w_{p j}\right)^{\prime}$, по данным столбца $\mathrm{PC}_{1}$ построили первую главную компоненту Y.

Вклад PC1 (Y1_1) в общую дисперсию индивидуальных показателей оценок максимален и в абсолютном выражении равен собственному числу $\lambda_{1}=130$, а в процентном $-81,71 \%$. Следовательно, первая главная компонента Y1_1 (PC1) может рассматриваться как интегральный. Остальными главными компонентами $\mathrm{PC}_{2}$ и $\mathrm{PC}_{3} \mathrm{c}$ незначительными вкладами в общую дисперсию можно пренебречь.

Вычислим значения Y1_1 для каждого из тридцати студентов экспериментальной группы по формуле (2). Результат вычислений приведен на рис. 4.

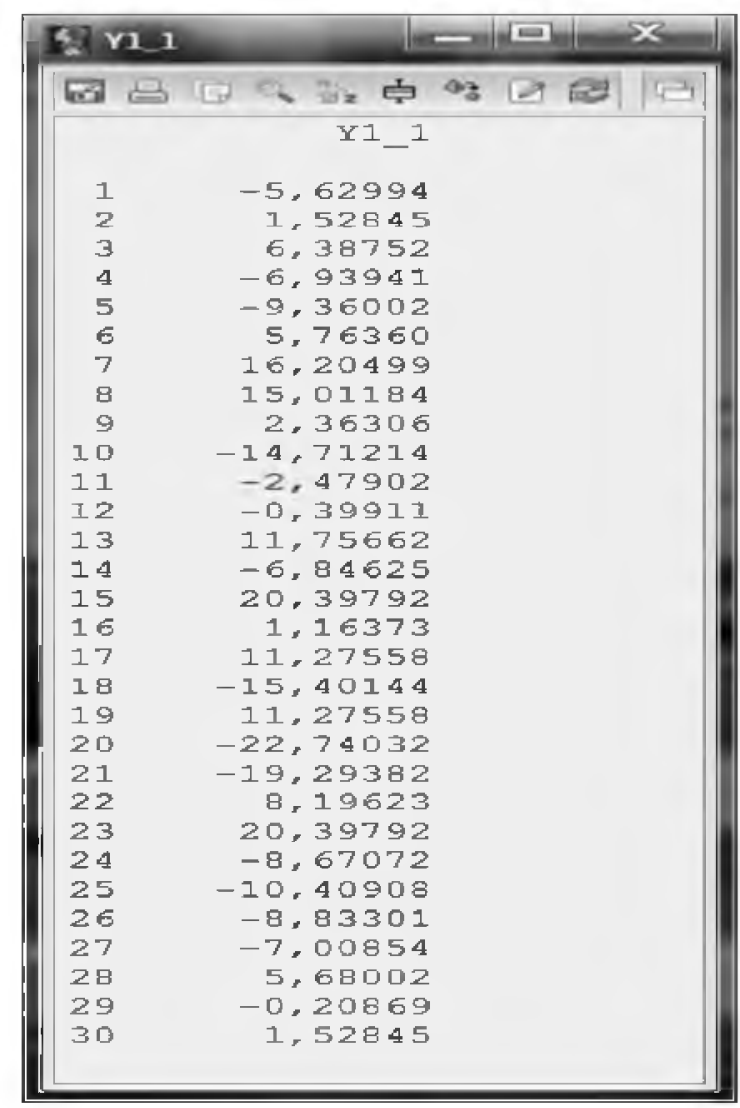

Рис. 4. Значение главной компоненты Y1_1

Интегральный показатель Y2_1 построим по пространству исходных признаков Semester_4, Semester_5, учитывая оценки за последние два семестра обучения английскому языку в Moodle. Реализация этого этапа в программной среде Gretl приведена на рис. 5. 


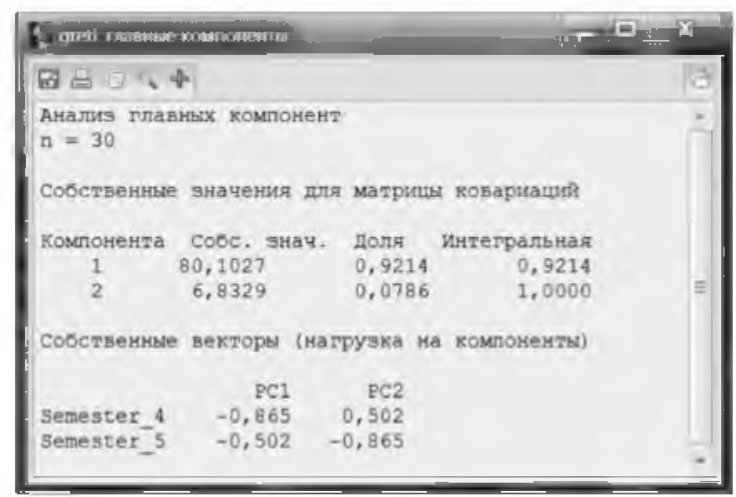

Рис. 5. Окно результатов моделирования интегрального показателя оценки по английскому языку после внедрения Moodle (последние два семестра обучения)

По результатам моделирования рис. 5 построим интегральный показатель оценки Y2_1 как первую главную компоненту (3):

$$
Y_{2 \_1}=-0,865 \cdot \text { Semester }_{4}-0,502 \cdot \text { Semester }_{5} .
$$

Это возможно, поскольку вклад $\mathrm{PC} 1$ (Y2_1) в общую дисперсию достаточен и составляет $80,1 \%$. Значения данной главной компоненты показаны на рис. 6

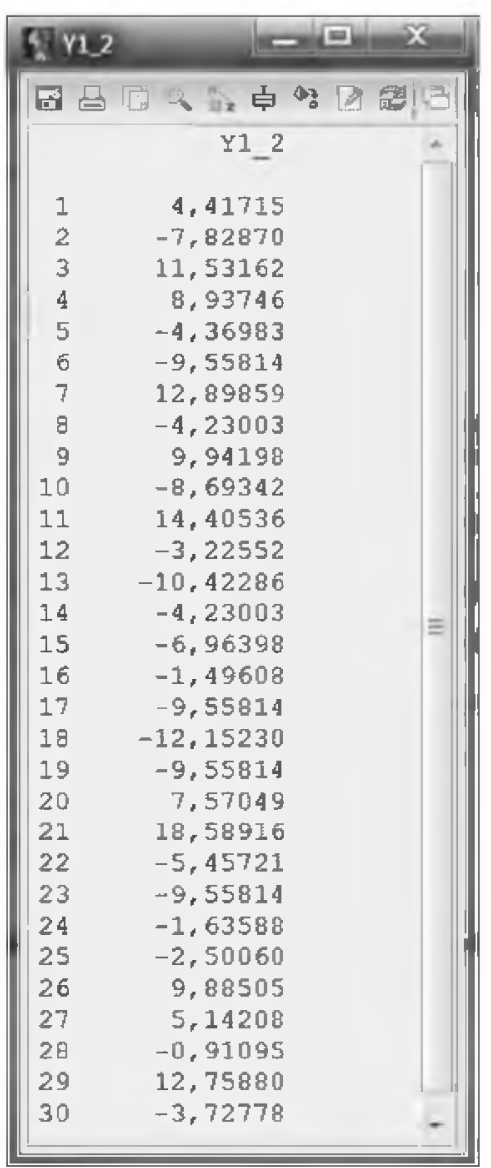

Рис. 6. Значение главной компоненты Y2_1 
Эman 3. Проанализируем динамику интегрального показателя оценки по английскому языку как разницу значений Y1_1 иY2_1 для худшего студента экспериментальной группы.

В первые три семестра лучшие результаты показали студенты №15 и №23, показав интегральный результат Y1_1 = 20,4 (рис. 5). Худший результат по данному показателю $(-22,74)$ показал студент № 20. После внедрения Moodle худший студент улучшил свой интегрированный результат (за два семестра) до 7,57, что может быть результатом использования электронного учебного курса по английскому языку. Этот вывод подкрепляется визуальным анализом коробчатой диаграммы на рис. 7 .

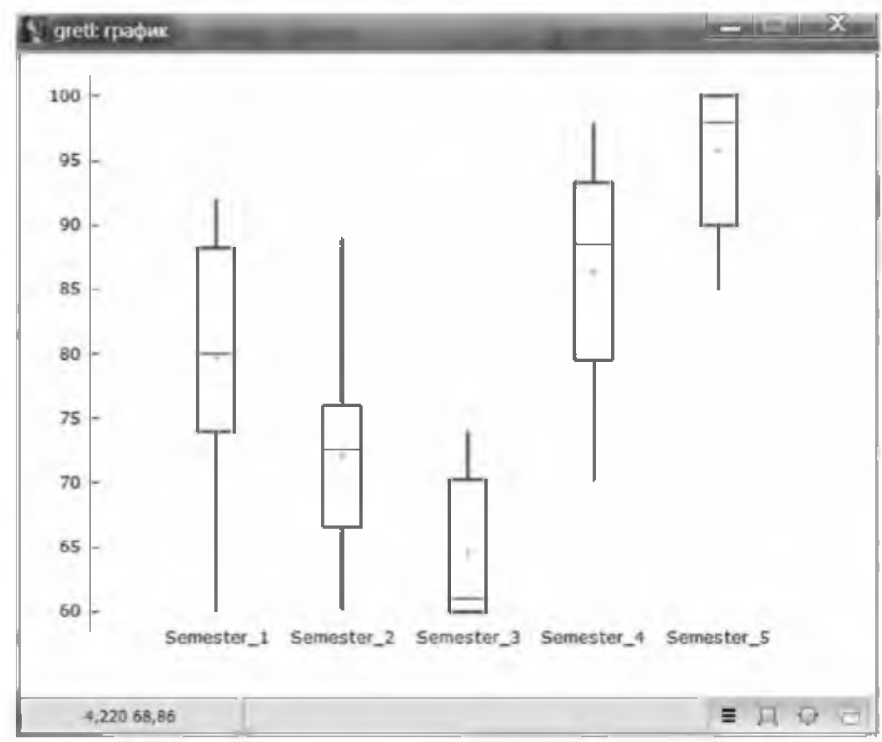

Рис. 7. Динамика среднесеместровой оценки экспериментальной группы

В первые три семестра средние арифметические за семестр, как и медианы, снижаются, а после внедрения электронного учебного кура имеют тенденцию к повышению.

Этап 4. Используем метод дисперсионного анализа, чтобы показать разницу средних оценок в третьем (непосредственно до Moodle) и в четвёртом (сразу после) семестре, рис. 8.

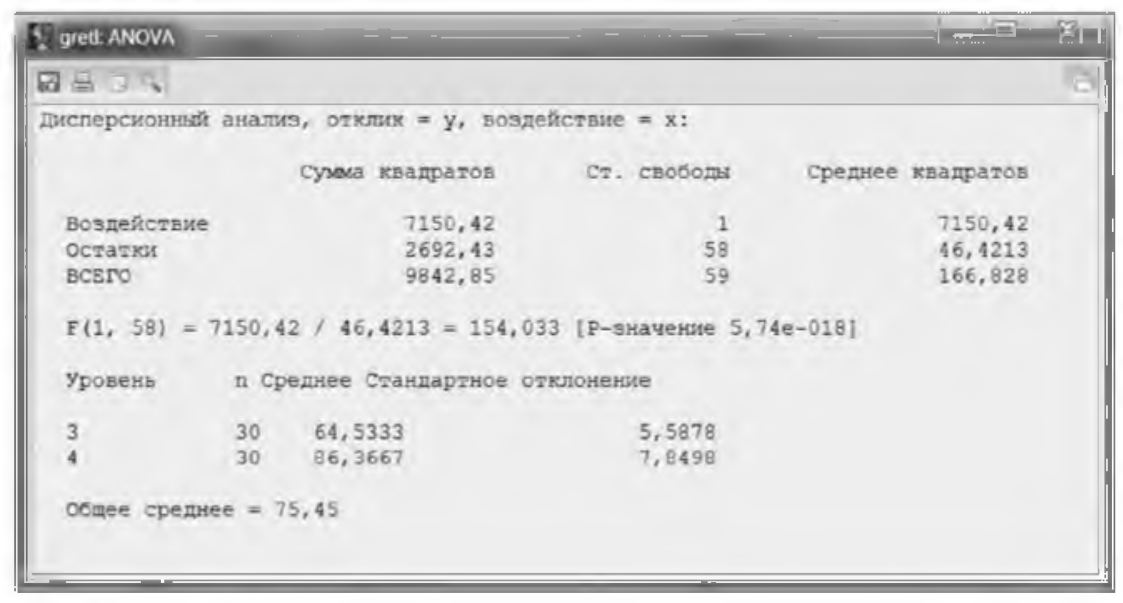

Рис. 8. Окно результатов моделирования с использованием инструментария дисперсионного анализа для экспериментальной группы 
Как показывает рис. 8 , поскольку р-значение $(5,74 \mathrm{e}-018)$ меньше уровня значимости 0,05 , то принимаем альтернативную гипотезу о том, что средние значения не равны при разных уровнях фактора $\mathrm{x}$, т. е. работа в информационной среде оказывает положительное воздействие на среднюю успеваемость студентов.

Этап 5. Проведём расчёты, аналогичные этапу 4 для контрольной группы, в обучении которой не использовались электронные учебные курсы.

Исходные данные показаны на рис. 9, а результаты моделирования на рис. 10.

\begin{tabular}{|c|c|c|c|}
\hline \multicolumn{4}{|c|}{ 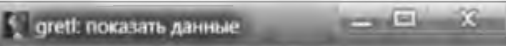 } \\
\hline \multicolumn{3}{|c|}{ 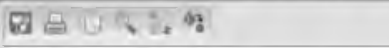 } & \multirow{2}{*}{$\frac{10}{4}$} \\
\hline & csemester_ 4 & csemester_3 & \\
\hline 1 & 60 & 65 & \\
\hline 2 & 65 & 66 & \\
\hline 3 & 69 & 70 & \\
\hline 4 & 61 & 60 & \\
\hline 5 & 60 & 60 & \\
\hline 6 & 60 & 60 & \\
\hline 7 & 80 & 85 & \\
\hline B & 70 & 70 & \\
\hline 9 & 63 & 67 & \\
\hline 10 & 60 & 60 & \\
\hline 11 & 61 & 61 & \\
\hline 12 & 60 & 60 & \\
\hline 13 & 82 & 90 & \\
\hline 14 & 60 & 60 & \\
\hline 15 & 74 & 74 & $\equiv$ \\
\hline 16 & 65 & 87 & \\
\hline 17 & 74 & 74 & \\
\hline 18 & 60 & 60 & \\
\hline 19 & 74 & 80 & \\
\hline 20 & 60 & 60 & \\
\hline 21 & 60 & 63 & \\
\hline 22 & 60 & 60 & \\
\hline 23 & 74 & 75 & \\
\hline 24 & 60 & 60 & \\
\hline 25 & 63 & 63 & \\
\hline 26 & 60 & 60 & \\
\hline 27 & 60 & 69 & \\
\hline 28 & 90 & 35 & \\
\hline 29 & 61 & 61 & \\
\hline 30 & 65 & 78 & -1 \\
\hline
\end{tabular}

Рис. 9. Набор исходных данных по контрольной группе в ПО Gretl (за семестр 3 и 4)

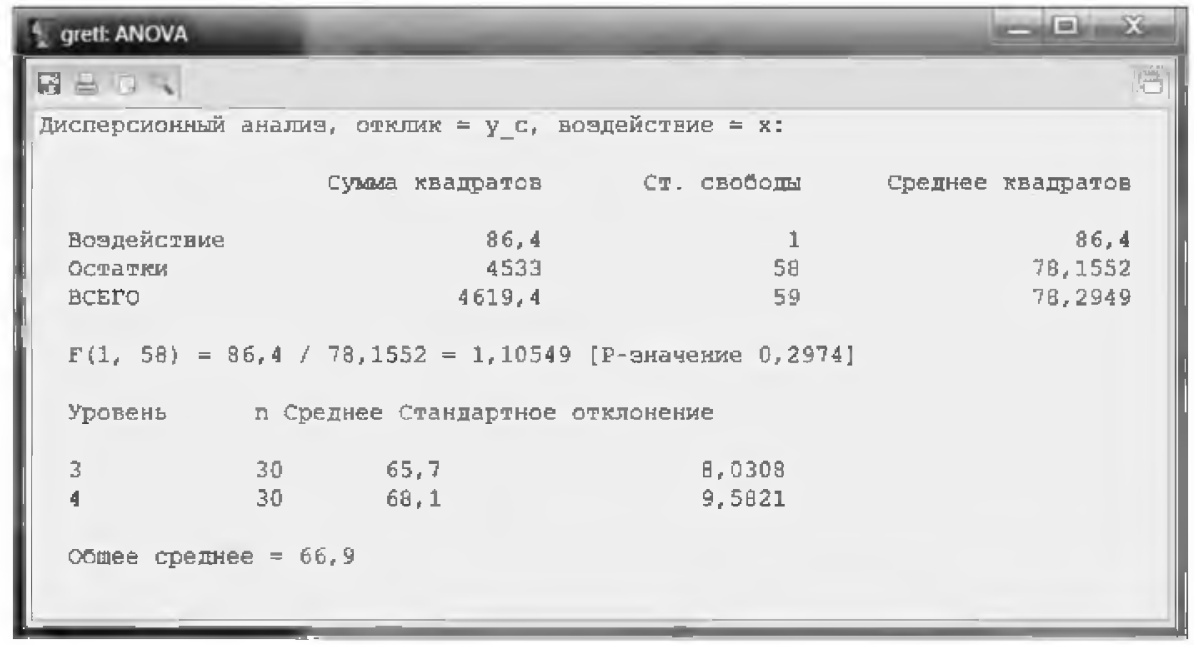

Рис. 10. Окно результатов моделирования, используя инструментарий дисперсионного анализа для контрольной группы 
Как показывает рис. 10 , поскольку р-значение $(0,2974)$ больше уровня значимости 0,05 , то принимаем нулевую гипотезу о том, что средние значения равны при разных уровнях фактора $\mathrm{x}$. Проиллюстрируем это на коробчатой диаграмме, рис. 11.

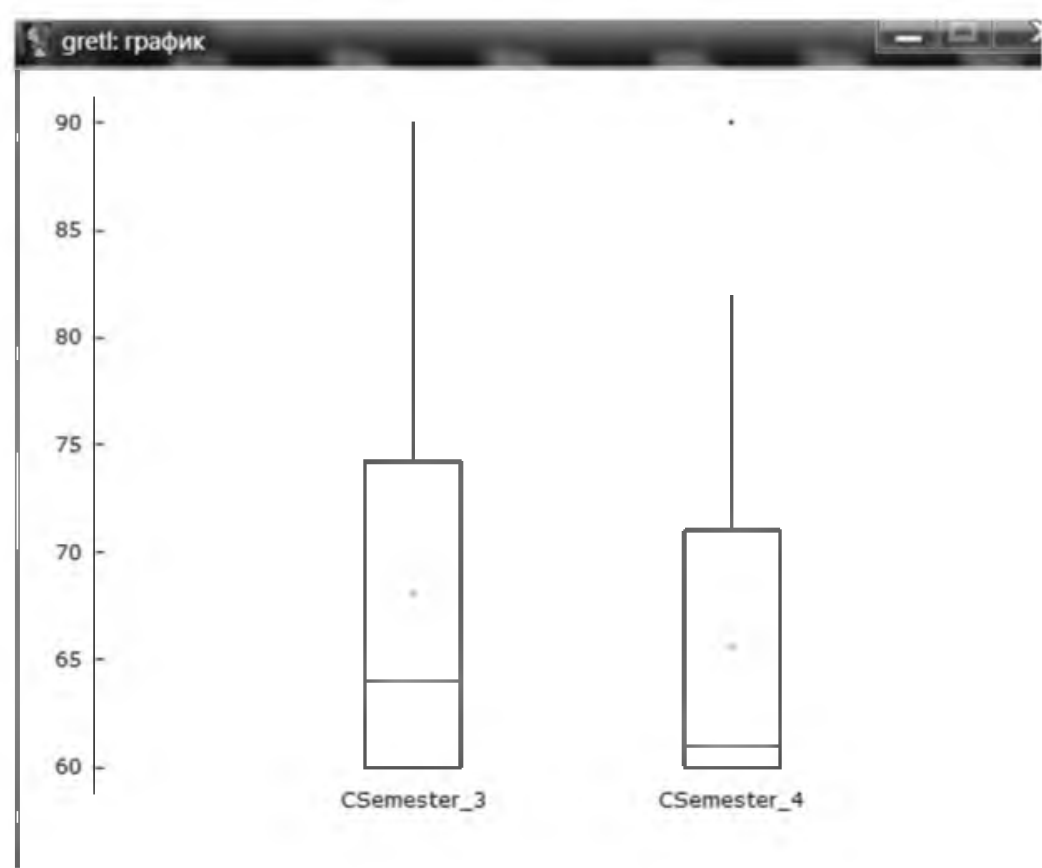

Рис. 11. Динамика среднесеместровой оценки контрольной группы

Из полученных на данном этапе вычислений (поскольку изменений в средней оценке не произошло для контрольной группы), можно сделать вывод, что при прочих постоянных факторах причиной повышения успеваемости в экспериментальной группе является использование цифровой образовательной среды. А именно использование электронных учебных курсов является фактором повышения успеваемости по английскому языку.

Заключение / Conclusion. Таким образом, нами описана организация и проанализированы результаты проведённого проверочного педагогического эксперимента по внедрению в образовательный процесс электронных учебных курсов по англоязычной подготовке студентов на базе Севастопольского государственного университета. Обработка полученных результатов методами главных компонент и дисперсионного анализа в ПО Gretl показали эффективность данного внедрения с точки зрения повышения успеваемости студентов по английскому языку.

\section{ЛИТЕРАТУРА И ИНТЕРНЕТ-РЕСУРСЫ}

1. Хикс Ч. Основные принципы планирования эксперимента. М.: Мир, 1967. 406 с.

2. Яковлев Е. В., Яковлева Н. О. Педагогическое исследование: содержание и представление результатов. Челябинск: РБИУ, 2010. 317 с.

3. Степанкин И. А. Особенности организации педагогического эксперимента // Современный учёный. 2017. № 6. C. 209-211.

4. Штофф В. А. Проблемы методологии научного познания. М.: Высшая школа, 1978. 271с.

5. Батищев Г. И. Педагогическое экспериментирование // Сов. педагогика. 1990. № 1.

6. Using gretl for Principles of Econometrics, 3rd Edition Version 1.01 Lee C. Adkins Professor of Economics Oklahoma State University. URL: http://www.learneconometrics.com/gretl.html

7. Куфель T. Эконометрика. Решение задач с применением пакета программ GRETL / T. Куфель. M.: Горячая линия - Телеком, 2007. 200 c. 


\section{REFERENCES AND INTERNET RESOURCES}

1. KHiks $\mathrm{CH}$. Osnovnye printsipy planirovaniya ehksperimenta (Basic principles of experiment planning). M.: Mir, 1967. $406 \mathrm{~s}$.

2. YAkovlev E. V., YAkovleva N. O. Pedagogicheskoe issledovanie: soderzhanie i predstavlenie rezul'tatov (Pedagogical research: content and presentation of results). CHelyabinsk: RBIU, 2010. $317 \mathrm{~s}$.

3. Stepankin I. A. Osobennosti organizatsii pedagogicheskogo ehksperimenta (Features of the organization of pedagogical experiment) // Sovremennyj uchyonyj. 2017. № 6. S. 209-211.

4. SHtoff V. A. Problemy metodologii nauchnogo poznaniya (Problems of methodology of scientific knowledge). M.: Vysshaya shkola, 1978. $271 \mathrm{~s}$.

5. Batishhev G. I. Pedagogicheskoe ehksperimentirovanie (Pedagogical experimentation) // Sov. pedagogika. 1990. № 1 .

6. Using gretl for Principles of Econometrics, 3rd Edition Version 1.01 Lee C. Adkins Professor of Economics Oklahoma State University. URL: http://www.learneconometrics.com/gretl.html

7. Kufel' T. EHkonometrika. Reshenie zadach s primeneniem paketa programm GRETL (Econometrics. Solving problems using the GRETL software package). M.: Goryachaya liniya-Telekom, 2007. $200 \mathrm{~s}$.

\section{СВЕДЕНИЯ ОБ АВТОРАХ}

Колесников Александр Михайловиц, доктор экономических наук, профессор кафедры экономики высокотехнологичных производств Санкт-Петербургского университета аэрокосмического приборостроения, г. Санкт-Петербург. E-mail: 9843039@mail.ru

Кокодей Татьяна Александровна, доктор экономических наук, профессор кафедры менеджмента и бизнес анализа Севастопольского государственного университета, г. Севастополь. E-mail: 9843039@mail.ru

Хитущенко Виталина Валерьевна, аспирант, Севастопольский государственный университет, г. Севастополь. E-mail: 9843039@mail.ru

\section{INFORMATION ABOUT AUTHORS}

Alexander Kolesnikov, Doctor of Economic Sciences, Professor of the Department of Economics of high-tech industries of St. Petersburg University of aerospace instrumentation, St. Petersburg. E-mail: 9843039@, mail.ru

Tatyana Kokodey, Doctor of Economic Sciences, Professor of management and business analysis Department of Sevastopol state University, Sevastopol. E-mail: 9843039@mail.ru

Vitalina Hitsenko, postgraduate student, Sevastopol state University, Sevastopol. E-mail: 9843039@mail.ru 